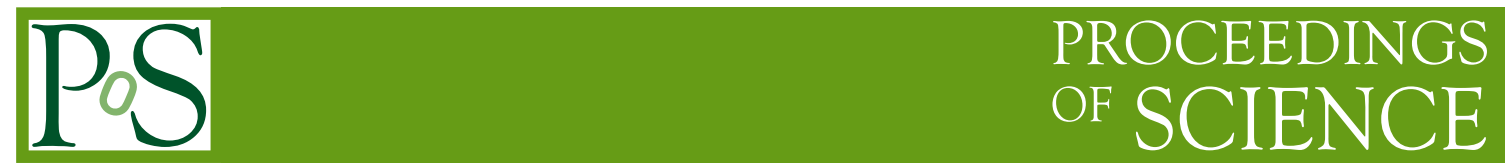

\title{
Study of 144ch HAPD for Aerogel RICH for Belle II
}

\author{
Shohei Nishida* \\ High Energy Accelerator Research Organization (KEK) \\ E-mail: shohei.nishida@kek.jp
}

\begin{abstract}
For the upgrade of the Belle detector (Belle-II) at the KEKB collider, we are developing a proximity focusing ring imaging Cherenkov detector using aerogel as a radiator. A 144 channel HAPD (Hybrid Avalanche Photo-Detector) is one of the candidate photo-detectors. We have performed a beam test with a $2 \mathrm{GeV}$ electron beam, and succeeded to detect a Cherenkov ring from the aerogel radiator. Tests under magnetic field and for neutron irradiation have also been performed.
\end{abstract}

International Workshop on New Photon Detectors PD09

June 24-26 2009

Shinshu University Matsumoto Japan

${ }^{*}$ Speaker. 


\section{Introduction}

The Belle experiment [1] with an asymmetric $e^{+}-e^{-}$collider KEKB [2] in KEK has made a critical contribution for the verification of the Kobayashi-Maskawa theory by observing the $C P$ violation in the $B$ meson system and precisely measuring the Cabibbo-Kobayashi-Maskawa (CKM) matrix elements. The experiment started in 1999 is planned to have a 3 year's shutdown for the upgrade of the KEKB accelerator and the Belle detector. The new experiment "Belle II" will focus on a search for New Physics using more than 50 times larger lumnosity provided by the "SuperKEKB" accelerator.

Presently, Belle is equipped with a threshold-type Cherenkov detector with aerogel radiator (ACC) [3] for $\pi / K$ separation above $1 \mathrm{GeV} / c$. However, ACC does not provide separation for high momentum particles around $4 \mathrm{GeV} / c$ in the forward end-cap region. For Belle II, we are therefore planing to use a ring imaging Cherenkov detector ( $\mathrm{RICH})$ with aerogel radiator [4] as a PID device in the forward region [5]. Our target is to provide around $5 \sigma \pi / K$ separation in the kinematical region ranging from $1 \mathrm{GeV} / c$ to $4 \mathrm{GeV} / c$. Due to space limitation, the detector must be of the proximity focusing type with an expansion gap of around $20 \mathrm{~cm}$. If we use aerogel with refractive index of $n=1.05$, the difference of Cherenkov angle between $\pi$ and $K$ is $23 \mathrm{mrad}$ at $4 \mathrm{GeV} / c$.

The photo-detector for the aerogel RICH need to satisfy the following criteria. The photodetector must be able to detect single photons, and have position sensitivy with a granularity of about $5 \times 5 \mathrm{~mm}^{2}$. The photon detection efficiency must be high enough for the reconstruct of the Cherenkov ring, which requires large effective area of the photo-detector. It also must be operational under $1.5 \mathrm{~T}$ magnetic field perpendicular to the photon detector plane. Finally, the photo-detector must cover the forward region in Belle II of around $4 \mathrm{~m}^{2}$, resulting in a total number of channels of about $10^{5}$.

One such candidate photo-detector is a 144 channel Hybrid Avalanche Photo-Detector (HAPD) developed in a collaboration with Hamamatsu Photonics K. K. (HPK).

\section{The 144 channel HAPD and its performance}

The structure of the 144 channel HAPD is shown in Fig. 1. Photo-electrons are accelerated by the high electric field over a voltage difference of around $10 \mathrm{kV}$ inside the vacuum tube, after which they enter the avalanche photo diode (APD), creating $O\left(10^{3}\right)$ electron-hole pairs. Amplification by the avalanche process inside the APD provides an additional gain of $O(10)$, resulting in the total gain of $10^{4}$ to $10^{5}$. Because of the simple acceleration of the photo-electrons in the first stage, the statistical fluctuation of the gain is rather small compared to conventional photo-multipliers.

In the counter under study, 4 APD chips are placed in a $2 \times 2$ array (Fig. 1b). Each chip is divided into 36 channels with a $4.9 \times 4.9 \mathrm{~mm}^{2}$ pixel size. The outer dimension of the HAPD is $73 \times 73 \mathrm{~mm}^{2}$, so the effective area is around $65 \%$. Maximum high voltage applied to the photocathode is $-8.5 \mathrm{kV}$, while the APD is biased with around $300 \mathrm{~V}$. The typical quauntum efficiency of the HAPD is $25 \%$.

Figure 2a shows the noise level of an HAPD when we vary the reverse bias voltage on the APD chips without applying high voltage to the photo-cathode. The noise level is estimated from the width of the pedestal for 4 channels that are located in the center of each APD chip. The 
(a)

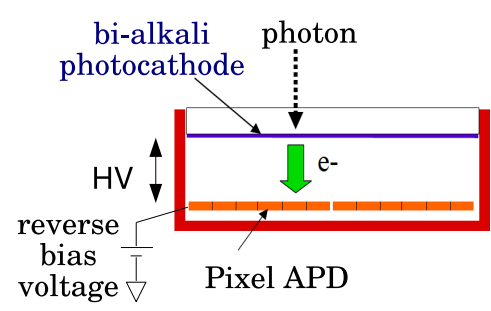

(b)

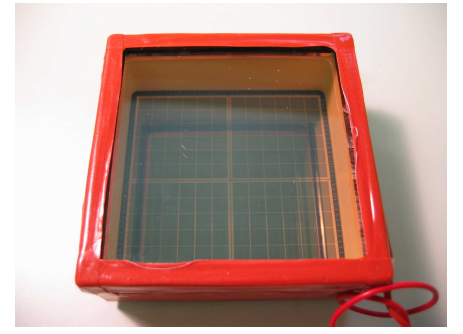

Figure 1: (a) Structure of a multi-anode HAPD. (b) 144ch multi-anode HAPD.

(a)

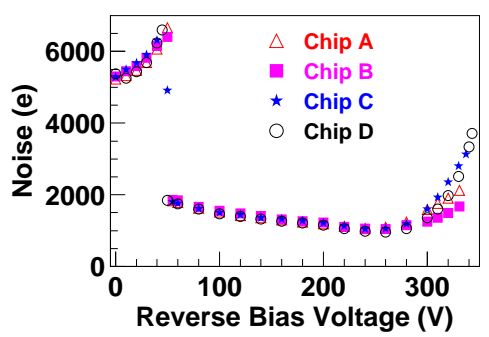

(b)

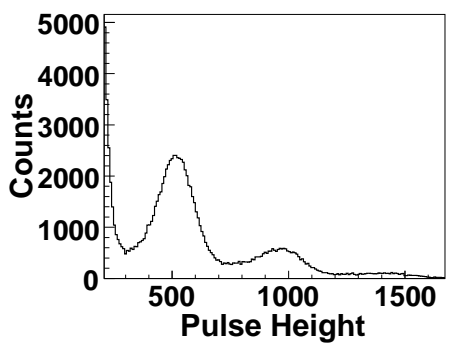

Figure 2: (a) Measured noise level of the HAPD with high voltage off. (b) ADC distribution of one channel of an HAPD; the large peak at the left edge is pedestal, while a peak in the left hand side corresponds to single photo-electrons.

noise suddenly gets smaller above $50 \mathrm{~V}$ of the bias voltage, where the full depletion is achieved. Figure $2 \mathrm{~b}$ shows the pulse height distribution for one channel when we apply high voltage of $-7 \mathrm{kV}$ to the photo-cathode. We use an LED as the light source, which is adjusted so that single photons are counted. We can see a clear separation among the pedestal and single photo-electron and two photo-electron peak.

\section{Beam Test of Aerogel RICH}

We performed two beam tests in March and June 2008 at the Fuji test beam line, where a $2 \mathrm{GeV}$ electron beam converted from Bremsstrahlung photons from the KEKB electron ring is available. The detector is composed of an aerogel radiator and an $2 \times 3$ array of HAPDs, whose photo-detection plane is parallel to the radiator face and at a distance of $20 \mathrm{~cm}$. Signals from the 6 HAPDs are read out by 48 ASICs that are develped by ourselves. We apply a high voltage of $-7 \mathrm{kV}$ to all HAPDs, and set the bias voltage of each APD at the avalanche gain of 40 . The thickness and refractive index of the typical aerogel tile are $2 \mathrm{~cm}$ and 1.045 , respectively.

Figure $3 \mathrm{a}$ shows the hit distribution at the photo-detection plane. A clear Cherenkov ring is observed. The Cherenkov angle distribution is shown in Fig. 3b. Typical number of photo-electron per track $\left(N_{\text {p.e. }}\right)$ is 4.6 , while the resolution $\left(\sigma_{\theta_{c}}\right)$ is $12.8 \mathrm{mrad}$, resulting in the single track resolution $\left(\sigma_{\theta_{c}} / N_{\text {p.e. }}\right)$ of $6.0 \mathrm{mrad}$. This corresponds to around $4.0 \sigma \pi / K$ separation at $4 \mathrm{GeV} / c^{2}$. 
(a)

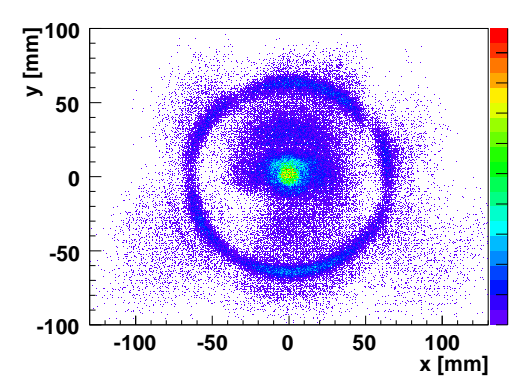

(b)

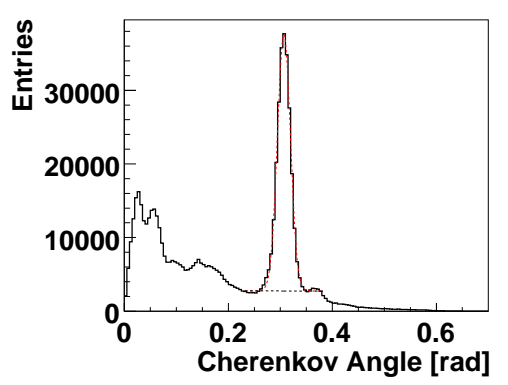

(c)

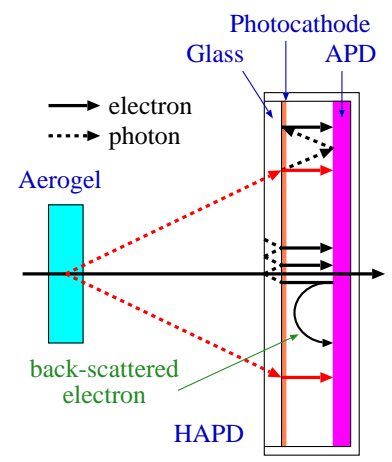

Figure 3: (a) Hit distribution at the photo-detection plane; for each hit, the difference between the beam position and the HAPD hit position is plotted. (b) Cherenkov angle distribution. (c) Explanation of the background contributions.

In Figure 3a, we find non-uniform background contribution inside the ring. We can also see an additonal ring outside of the Cherenkov ring. After several measurements in a test bench and the beam test, we find these contributions can be explained as shown in Fig. 3c. The reflection of the Cherenkov photon from the beam generated inside the glass produces the structure inside the ring. This contribution can be reduced by reducing the thickness of the HAPD (presently $4 \mathrm{~mm}$ ). There is also contribution of the back-scattering of the photo-electrons. The Cherenkov photons that pass through the photo-cathode may be reflected at the APD, reach the photo-cathode and be converted to a photo-electron, making the outer ring.

\section{Test under the Magnetic Field}

HAPDs must be operated under 1.5 T magnetic field that is perpendicular to the photon detector plane. In the magnetic field, the trajectories of the electrons inside the HAPD are coiled around the lines of the magnetic force, and the back-scattered electrons from the APD fall very near to the location where the initial photo-electron hits. Therefore, in magnetic field, we expect smaller contribution from back-scattered electrons, to the background distribution inside the Cherenkov ring. Similarly, the magnetic field is supposed to reduce the inefficiency or cross-talks seen in the channels near the edge of a HAPD, which is considered to be due to a distortion of electric field inside the tube.

We have performed a test of the HAPD in magnetic field in order to confirm the operation of the HAPD and the side effects of the magnetic field. Figure 4 shows the hit distributions of the channels for the pixel at the edge of a HAPD and for the next pixel when we perform an onedimensional scan using a LED with small light spot. When we apply no magnetic field, the edge channel is sensitive to only a part of the region that correspond to the APD pixel at the edge. On the other hand, the next channel has some sensitive location outside of the APD pixel. We cannot see these inefficiencies and cross-talks under $1.5 \mathrm{~T}$ magnetic field.

When we vary the position of the light spot and monitor the output of one channel, we find the signal when the light spot is located in the APD pixel that corresponds to the monitored channel. 
(a)

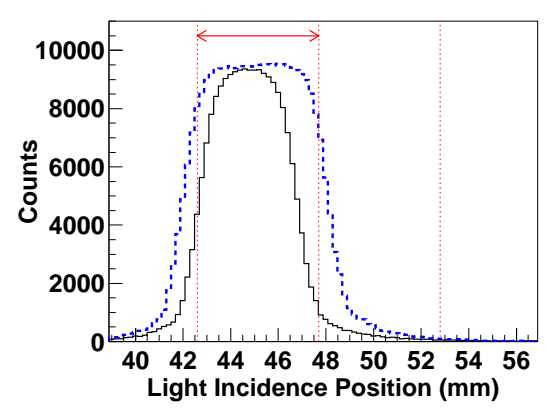

(b)

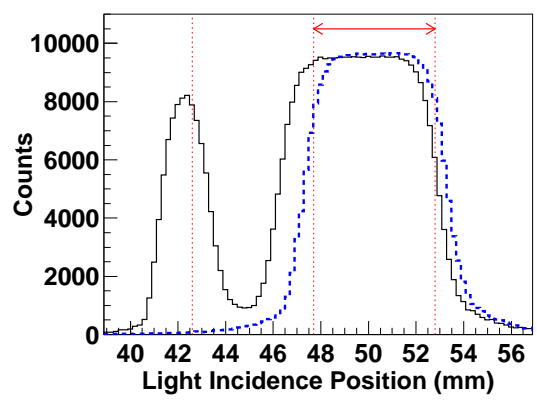

Figure 4: Light incidence position dependence of the hit distributions for (a) pixel at the edge (b) next pixel. The black solid and blue dashed histograms show the distribution at $0 \mathrm{~T}$ and $1.5 \mathrm{~T}$ magnetic field, respectively. The vertical red dotted lines show boundaries of the APD pixels, and the red arrow indicates the sensitive region of the pixel.

However, small amount of hits exist even when the light spot is far from the pixel. The maximum distance between the light spot and the pixel with hits are around $40 \mathrm{~mm}$ and $15 \mathrm{~mm}$, respectively for $0 \mathrm{~T}$ and $1.5 \mathrm{~T}$ magnetic field. The former is consistent with the maximum distance that backscattered electrons can reach without reaching the photo-cathode plane that is $20 \mathrm{~mm}$ away from the APDs. In the magnetic field, the back-scattered electron can travel less than $1 \mathrm{~mm}$, so we consider the remaining contribution is due to photon scattering at the APDs. From this measurement, we conclude that some of the background distribution inside of the Cherenkov ring (Fig 3c) is due to back-scattered electrons and that their effects are reduced in the magnetic field.

\section{Radiation Hardness}

One of the remaining issues in HAPDs is radiation hardness, especially the tolerance for neutrons. In Belle-II, we expect $10^{11} \mathrm{~cm}^{-2}$ neutrons per year in the endcap region where the aerogel RICH is equipped. Considering the 10 years' operation, photo-detectors must be tolerant around $10^{12} \mathrm{~cm}^{-2}$ neutrons.

We have performed neutron irradiation test at the Yayoi, fast neutron source reactor of the University of Tokyo, in Tokai village. We have irradiated $1 \times 10^{10} \mathrm{~cm}^{-2}$ and $5 \times 10^{11} \mathrm{~cm}^{-2}$ neutrons to one HAPD, and measured the leakage current and pulse height distributions with light source.

Figure 5 shows the pulse height distributions before and after neutron irradiation for one channel. After $5 \times 10^{11} \mathrm{~cm}^{-2}$, noise gets increased and $S / N$ clearly gets worse, in all the channels, though there is channel dependence. The leak current is also increased by factor of 3 . This result have raised some concern about the radiation hardness of HAPD to neutrons. We are planning to test more samples in future.

\section{Conclusion}

We have developed a 144 channel HAPD in a collaboration with HPK. HAPDs show good performance to detect single photons, and a Cherenkov ring is clearly observed in the beam test of 
(a)

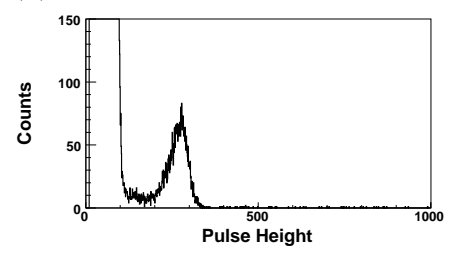

(b)

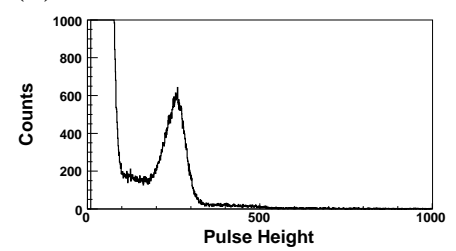

(d)

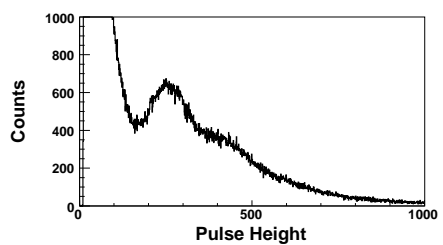

Figure 5: Pulse height distribution (a) before irradiation (b) after $1 \times 10^{10} \mathrm{~cm}^{-2}$ neutrons irradiation (c) after $5 \times 10^{11} \mathrm{~cm}^{-2}$ neutrons irradiation. The measurement of (c) is done 20 days after irradiation.

prototype aerogel RICH using HAPDs. We have confirmed the operation under magnetic field and the reduction of the contribution from back-scattered electrons in the tube. We have also tested the effect of neutron irradiation to a HAPD, but haven't obtained a conclusive result about radiation tolerrance.

We would like to thank the Belle collaboration for its help in this project. We would like to thank Hamamatsu Photonics K.K. for a good collaboration in the development of the HAPD. This work was supported by the Ministry of Education, Science and Culture of Japan.

\section{References}

[1] A. Abashian et al. (Belle Collaboration), Nucl. Instr. and Meth. A479 (2002) 117.

[2] S. Kurokawa and E. Kikutani, Nucl. Instrum. Meth., A499, 1 (2003), and other papers included in this Volume.

[3] T. Iijima et al., Nucl. Instr. and Meth. A453 (2000) 321.

[4] T. Matsumoto et al., Nucl. Instrum. Meth. A521 (2004) 367; T. Iijima et al., Nucl. Instrum. Meth. A548 (2005) 383.

[5] K. Abe et al., "Letter of Intent for KEK Super B Factory", KEK report 2004-04, available at http://superb.kek.jp/. 